\title{
Influence of the vacuum resin process, on the ballistic behaviour of lightweight armouring solutions
}

\author{
M. Lefebvre ${ }^{1,2}$, F. Boussu ${ }^{1,2}$, D. Coutellier ${ }^{1,3}$, and D. Vallee ${ }^{4}$ \\ ${ }^{1}$ Univ. Lille Nord de France, 59000 Lille, France \\ 2 ENSAIT, GEMTEX, 59100 Roubaix, France \\ ${ }^{3}$ UVHC, LAMIH FRE CNRS 3304, 59300 Valenciennes, France \\ ${ }^{4}$ NEXTER SYSTEMS Pôle Blindages, 11 allée des Marronniers, 78022 Versailles, France
}

\begin{abstract}
The armour of vehicles against conventional threats is mainly composed with steel or aluminium panels. Efficient heavy solutions exist, but the involved industries require new lightweight structures. Moreover, unconventional threats as IEDs (Improvised Explosive Devices) may cause severe damages on these structural and protective panel solutions. Thus, combination of aluminium or steel plates with textile composite structures used as a backing, leads to the mass reduction and better performance under delamination behaviour against these new threats. This paper is a part of a study dealing with the impact behaviour of three warp interlocks weaving structures under Fragment Simulating Projectile (FSP) impact. During this research, several parameters has being studied as the influence of the yarns insertions [1-4], the degradation of the yarns during the weaving process [5-7], and the influence of the resin rate on the ballistic behaviour. The resin rate inside composite materials is dependant on the final application. In ballistic protection, we need to control the resin rate in order to have a deformable structure in order to absorb the maximum of energy. However, with the warp interlocks weaving structure, the yarns insertions induce empty spaces between the yarns where the resin takes place without being evacuated. The resin rate inside the warp interlocks structures is in the most of cases less than 50\%, which lead to have brittle and hard material during the impact. Contrary to interlocks structures, the existing protection based on prepreg structure have a high fibres ratio around $88 \%$ of weight. That leads to have the best ballistic properties during the impact and good deformability of the structure.

The aim of this paper is to evaluate the influence of the resin rate on the ballistic results of the composites materials. For that, we have chosen two kinds of warp interlocks fabrics which were infused with epoxy resin following two processes. The first is a classical vacuum resin infusion; the second used a press in order to reach a resin ratio near to the existing protection. The existing protection is a prepreg structure with a fibre content of $88 \%$. It has been revealed that a resin rate less than $35 \%$ inside the warp interlocks composite material leads to have equivalent ballistics performances than existing protection.
\end{abstract}

\section{Introduction}

The ballistic protection area concerns a large area which includes the soft protection: as bullet proof vest for human body or helmets for soldiers, the hard protection: as ballistics vest for soldier or armour of vehicles located.... The ballistic threat is always changing, continuously improved and protections need to be enhanced. In hard ballistic, the main protection used is composed of metallic materials. For that, high strength steels, aluminium alloys and titanium alloys are predominantly used as armour due to the combination of high strength, good toughness along with good formability, and good ballistic performance. However, complete metallic material solutions are rather heavy. This fact has inspired researchers to develop, with the same or upper performance, lighter materials and different material combinations.

The advent of ultra high modulus polyethylene and aramid fibres has enhanced the effectiveness of fabric armour and generated interest in ballistic performance of composite armours made from these fibres. Layered structures are generally used in armoured systems which consist in two main parts: front layer and support layer. Front layers are made from metal or ceramic materials. They aim is to break the nose shape of the projectile and then reduce its ballistic efficiency. The back layers are assigned to absorb the remaining kinetic energy of the projectile $[8,9]$. Composite materials were established as materials for construction in virtually every sector of industry. The benefits obtained from the combined properties of individual constituent materials make an attractive proposition with excellent strength and stiffness to weight ratio, good corrosion resistance, etc. They also offer a unique capability to design a material which accurately addresses functionality requirements which is free of compromises usually associated with traditional isotropic materials. All users' requirement is to have good performance and mass production with low cost, but these properties are generally not possible at the same time [10]. However, the poor impact resistance of laminated composites has hindered their applications in many engineering structures. Therefore, many studies have been carried out to help understanding the impact response of composite materials and structures and improve the composite design. Based on interlacing fibre yarns, some woven composites have been found to outperform their laminated counterparts in impact resistance. Some articles concerning the ballistic response of woven composites may be found in the literature. Thus, Siow and Shim [11] have investigated the ballistic response of woven composites made of carbon fabrics and an epoxy matrix. Mechanical properties of the damaged composites have also been examined. Davies et al. [12] have studied 


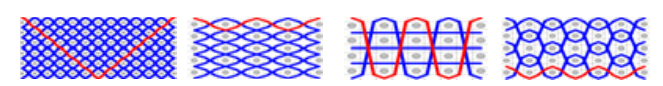

Fig. 1. Illustration of the classification of warp interlocks [19].

the impact-induced damage and residual strengths of thick glass/polyester woven composites. Rydin et al. [13] have addressed the influence of low velocity impact testing of woven and nonwoven composites. They have concluded that impact velocity only affects the initial part of load curve. Wu and Chang [14] have investigated the effect of loading rate on the E-glass/epoxy woven composites subjected to quasi-static load. They have found that fibre breakage throughout the layers of the composites absorb a significant amount of impact energy compared to other damage mechanisms. Kim and Sham [15] have presented an overview of fracture behaviour and delamination failure mechanism of woven composites subjected to impact loading. In their study, the advantages of using woven fabrics over cross-ply laminates based on microstructure property analysis have also been presented.

Traditional laminated, woven and braided composite structures are inherently susceptible to inter-layer delamination due to their micro-structural geometry and weak inter-laminar bonds. Specifically, in hard armour applications, composites should possess a high area density, superior delamination resistance and high fracture toughness. Three-dimensional (3D) woven composites are a class of materials that encompass all of the above mentioned properties. The micro-structural geometry of 3D woven composites is characterized by several layers of [0/ 90] laminates 'bound' together with the use of warp weavers or Z-yarns. This process imparts suitable mechanic characteristics such as high in-plane and through-thickness stiffness and strength, superior delamination resistance and high fracture toughness. Cox and al. [16] have investigated various aspects of the response of $3 \mathrm{D}$ woven composites in different environments. Elastic properties, strength, ductility and fatigue life of 3D woven composites were experimentally determined to be highly sensitive to tow waviness.

Although the third yarns insertion allows having good interlaminar properties; he also creates empty area inside the structure due to the interlacing of the yarns and the crimp created [17]. During the infusion process, the resin could be more easily inserted inside these empty spaces, leading to have a final composite material with high resin rate. However, in ballistic protection, we need to benefit on the role of high performances fibres, but also the geometrical structure, instead of the resin which is principally present for the consolidation of the structure to give additional properties.

Thus, we have to take care of the resin infusion process in order to have good ballistic behaviour and results. In order to quantify the amount of resin rate needed to have good performances we have chosen to work with two kinds of warp interlocks structures, which have shown their efficiency during previous study [1-8]. These two structures are infused under two different processes, one is a classical vacuum resin infusion, the other is an infusion coupled with a press in order to control the amount of resin. After that, we determine the limit speed of protection for
Table 1. Weaving parameters of the two warp interlocks structures.

\begin{tabular}{|l|c|c|}
\hline & « Ortho $»$ & $\ll \mathrm{TtT} »$ \\
\hline Warp density (ends/cm) & 20 & 20 \\
\hline Weft density (picks/cm) & $40<x<60$ & $30<x<50$ \\
\hline Type of fibres & Vectran $®$ & Vectran $®$ \\
\hline
\end{tabular}

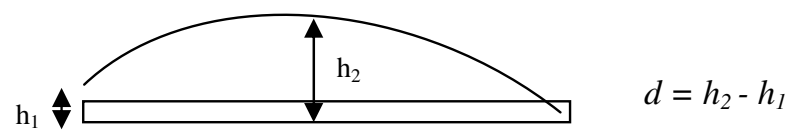

Fig. 2. Determination of the back-face deformation after the impact.

these two composites materials, located at the back-face of a metallic material. A comparison with existing protection is also made to determine the potential of warp interlocks structures.

\section{Context of the study}

\subsection{The weaving structures}

This study is a part of a three years research project on the warp interlocks structures used for composites material in ballistic protection. This project deals with different subject as the influence of geometrical warp interlocks weaving on the ballistic performance of the composites materials, the influence of the degradation of the yarns during the weaving process, the influence of the resin rate on the behaviour of the final composite material under FSP impact.

The multilayer woven fabrics as warp interlocks are used as fibrous reinforcement inside composite material where high mechanical properties are needed. Descriptions of the many advantages are given by J. Hu [18]. Although these structures are typically more expensive than 2D fabrics, they reduce labour, have higher performances and improve process efficiency result in overall cost saving in a variety of applications. When we compare the cost per square meter of finished composite structure, the 3D woven fabric reinforcements consistently outperform traditional 2D materials. These advantages could be completed with these following studies. Indeed $\mathrm{Hu}$ et al [18] have mentioned that the 3D structures have an increase of compression and flexural strength after impact compared to 2D laminates and a decrease of the delaminated area. Indeed 2D woven fabric are generally anisotropic, have poor in plane shear resistance and have lower Young modulus value than the fibre materials due to the existence of crimp and yarns friction. Moreover, the major disadvantage of $2 \mathrm{D}$ is their low through the thickness mechanical properties because of the lack of Z-direction fibres. The two dimensional arrangement of fibres provides very little stiffness and strength in the thickness direction, leading to delamination of a structure during an impact. 
Table 2. Characteristics of the warp interlocks structures after the two resin process.

\begin{tabular}{|l|c|c|c|c|}
\hline & Ortho $1 »$ & «tT $1 »$ & 《Ortho 2 & 《tT 2 » \\
\hline Warp density (ends/cm) & 20 & 20 & 20 & 20 \\
\hline Type of infusion & Vacuum infusion & Vacuum infusion & $\begin{array}{c}\text { Vacuum infusion } \\
\text { with press }\end{array}$ & $\begin{array}{c}\text { Vacuum infusion } \\
\text { with press }\end{array}$ \\
\hline Fibre rate $(\% \mathrm{wt})$ & $30<\mathrm{x}<40$ & $\mathrm{x} \leq 50$ & $\mathrm{x} \leq 70$ & $70<\mathrm{x}<80$ \\
\hline $\begin{array}{l}\text { Loss of thickness }(\%) \\
\text { before and after resin infusion }\end{array}$ & 8 & 23 & 36 & 53 \\
\hline
\end{tabular}

Table 3. Characteristics of the warp interlocks structures after the impact tests.

\begin{tabular}{|c|c|c|c|c|}
\hline & 《 Ortho $1 »$ & $\ll \mathrm{TtT} 1 »$ & 《 Ortho $2 »$ & $\ll \mathrm{TtT} 2 »$ \\
\hline Type of infusion & Vacuum infusion & Vacuum infusion & $\begin{array}{c}\text { Vacuum infusion } \\
\text { with press }\end{array}$ & $\begin{array}{c}\text { Vacuum infusion } \\
\text { with press }\end{array}$ \\
\hline Fibre rate $(\% \mathrm{wt})$ & $30<x<40$ & $\mathrm{x} \leq 50$ & $\mathrm{x} \leq 70$ & $70<x<80$ \\
\hline Impact ratio & 72400 & 101900 & 97300 & 127200 \\
\hline$\%$ of benefit to use Vacuum infusion with press & & & 26 & 20 \\
\hline Deformation $(\mathrm{mm})$ case of non penetration & 22,2 & 24,4 & 50,1 & 51,5 \\
\hline$\%$ of benefit to use Vacuum infusion with press & & & 56 & 53 \\
\hline
\end{tabular}

The 3D warp interlocks woven structures can be classified into "angle interlock" and "orthogonal interlock" bonding according to the orientation of binders, or "through the thickness" and "layer to layer" bonding if the penetration depth of binders is concerned. Hu et al [12] proposed a classification with four groups of warp interlocks ie angle interlock/through-thickness bonding (A/T), angle interlock/layer to layer bonding $(\mathrm{A} / \mathrm{L})$, orthogonal interlock/through thickness bonding $(\mathrm{O} / \mathrm{T})$ and orthogonal interlock/layer to layer bonding $(\mathrm{O} / \mathrm{L})$ [19] (Figure 1).

For our project, three warp interlocks geometries have been produced and tested with different layers, two yarns densities and two kinds of yarns: an aromatic polyester fibre call Vectran $($ and the Kevlar $($ p para-aramid fibre. For this paper, two warp interlocks structures are considered, corresponding to the $\mathrm{A} / \mathrm{T}$ and $\mathrm{O} / \mathrm{T}$ bonding called respectively "TtT" and "Ortho". To compare our structures, we have also work with existing protection; prepreg structures composed of different plies. Yarns insertion in these two warp interlocks structures plays an important role to determine the mechanical properties of the structure.

In A/T structure, all warp yarns are going through the thickness of the fabric with a certain angle of inclination. This yarn placement could induce a lot of interested properties especially for the deformability of the structure during the impact. Moreover we can predict that, during an impact loading, all the warp yarns which are directly in contact with the projectile will distribute the mechanical stresses along their neighboured yarns. Indeed for this type of architecture, the warp and weft yarns have a lot of crossing for each weft rows and each layer.
Table 4. Mechanical properties of Kevlar@ and Vectran $®$ fibres [1-4].

\begin{tabular}{|c|c|c|c|c|}
\hline & $\begin{array}{l}\text { Linear } \\
\text { density } \\
(\mathrm{dTex})\end{array}$ & $\begin{array}{l}\text { Breaking } \\
\text { tenacity } \\
(\mathrm{cN} / \text { tex })\end{array}$ & $\begin{array}{c}\text { Breaking } \\
\text { elongation } \\
(\%)\end{array}$ & $\begin{array}{c}\text { Young } \\
\text { Modulus } \\
(\mathrm{GPa})\end{array}$ \\
\hline $\begin{array}{l}\text { Kevlar® } \\
\text { fibres }\end{array}$ & 3300 & 180 & 4,7 & 56 \\
\hline $\begin{array}{l}\text { Vectran } \AA \\
\text { fibre }\end{array}$ & $2 \times 1650$ & 210 & 4,7 & 63 \\
\hline
\end{tabular}

$>$ In $\mathrm{O} / \mathrm{T}$ structure, most of the warp yarns are kept straight and two warp yarns are passing through the thickness. During the impact, we can assume that the longitudinal yarns can receive the strain and resist against the load because they will react simultaneously and contribute to their full strength to that direction. Moreover when the ballistic panel will be stretched, the straight warp yarns would react to low elongation.

The different parameters for all the structures are described on the Table 1, where we find the type of warp interlocks structures, the warp and weft densities respectively expressed in ends/cm and picks/cm and the type of fibres used.

\subsection{The resin process infusion}

Two different resin infusions have been used to produce the final composite material. The first one is a classically vacuum resin process where the fibrous material is put 
Table 5. Comparison with existing protections of the four warp interlocks structures.

\begin{tabular}{|l|c|c|c|c|c|}
\hline & - Ortho $1 »$ & $\ll \mathrm{TtT} 1 »$ & 《Ortho 2» & 《tT $2 »$ & 15 plies of prepreg \\
\hline Fibre rate $(\% \mathrm{wt})$ & $30<\mathrm{x}<40$ & $\mathrm{x} \leq 50$ & $\mathrm{x} \leq 70$ & $70<\mathrm{x}<80$ & 88 \\
\hline Impact ratio & 72400 & 101900 & 97300 & 127200 & 132500 \\
\hline Deformation $(\mathrm{mm})$ case of non penetration & 22,2 & 24,4 & 50,1 & 51,5 & 54,1 \\
\hline
\end{tabular}

under a vacuum bagging. The resin is introduced and evacuated through a pipe with a void pressure around 0,1 $\mathrm{MPa}$. This process is well used when the structures infused have different thickness and weight. We can also control the resin progression inside the material and stopped the infusion when we want. However, the lack of pressure does not allow an ideal evacuation of resin leading to have a high rate.

The second used the autoclave technique. After the resin was introduced with a vacuum process, the structure was pressed with a high pressure (pressure is more than $0,6 \mathrm{MPa}$ ) in order to obtain a fibrous material with a minimal rate of resin. This process has the advantage to evacuate a maximum of resin leading to have final composite material with high rate of fibres (around 70\%). However the pressure applied, have maybe some influence on the geometrical structures after the infusion leading to have different behaviour during the impact. This point was not yet checked. Tomography results can help to make a conclusion on the use of this process and correlate the impact results with the geometrical structures obtained.

\section{Impact tests}

Impact tests are performed with fragment simulating projectile (FSP). The aim of theses different tests is to obtain the limit of velocity (Vlp) for each structure. The Vlp is measured with the average of non perforating projectile velocity and the velocity of the perforating projectile. For that, we used three ballistic plates and for each of them we have made one shot. Thus, we are not dependant of boundary conditions especially the deformation of the plate and the yarns after the first shooting tests. The impact velocities are between 500 to $1000 \mathrm{~m} / \mathrm{s}$. The impact velocity was adjusted for each structure depending on the final thickness of the ballistic plate and the final area density.

These impact results are different from a structure to another. The results depend on the kind of structures, the resin rate and the deformation way. Moreover the tested structures are very different concerning the area density. We cannot compare the Vlp of a structure with low density and for a structure with high density and conclude on the ballistic performance for each of them. In order to have a precise comparison, we have introduced an "Impact ratio" taking into account the Vlp and the area density for each plate. This ratio helps us to compare the ballistic performance of the structure at the same area density.

It could be also interested to observe the back-face deformation (called $d$ in $\mathrm{mm}$ ) of the composite materials after the impact, for the cases where the FSP were stopped.
These deformations were calculated taking into account the thickness of composite material before the impact $\left(h_{1}\right)$, and the thickness of the maximal conical deformation after the impact $\left(h_{2}\right)$.

\section{Results and discussions}

The results are summarized on Tables 2 and 3, where the fibre rates the thicknesses of the structures, the impact ratio and the back-face deformation are mentioned. . The table 2 shows that the "TtT" fabric is more deformable than "Ortho" leading to have more compaction during the infusion process. However, we observe a better back-face deformation for the "TtT" after the impact with a best Impact ratio.

The geometrical structure of interlocks fabrics leads to have little fibres ratio inside composite material. The empty areas are filled by the resin leading to have more resin than fibres for the first infusion process. Contrary to the infusion with less pressure, the second one is more efficiency because of the high fibres rate obtained after infusion (around $70 \%$ for each case). However, when we compare the thicknesses of the different structures, we can remark that the high pressure has crushed the fabric with a loss of thicknesses up to $30 \%$ for the warp interlock 2 . The compaction is more visible in the case of "TtT 1 " and "TtT 2" structures which are more deformable owing to the diagonal insertion of the yarns. These observations have to be completed with another test as tomography observation.

As we can see on the table 3 , the vacuum resin process using the high pressure seems to have a real influence on the final ballistic properties of the material. Indeed, the impact ratio rises up $20 \%$ with this process for the both structures, as the back-face deformation with an increase of $50 \%$. The increase of back-face deformation, shows that the structures have absorbed a lot of energy during

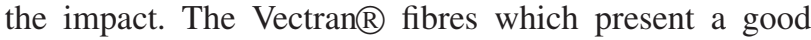
elongation at break $(4,7 \%)$ for a high tenacity at break allow a good deformation of the structure without damage the fibres and lead to have a good Impact ratio.

It's also important to compare the warp interlocks structures with existing protection in order to evaluate the potential of our fabrics. The existing protections are determined by the actual protection use like prepreg fabric. The existing protections are made with Kevlar@ fibres, but the mechanical properties of Kevlar $\AA$ and Vectran $®$ fibres are similar, as we can see on the table 4 .

The comparison with existing protection show that the prepreg structures predominate with an impact ratio up to the warp interlocks structures infused with a low pressure 


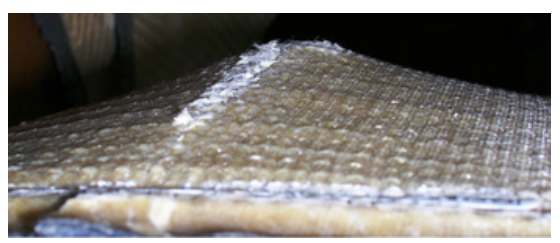

Fig. 3. Illustration of the back-face deformation after impact case of "Ortho1".

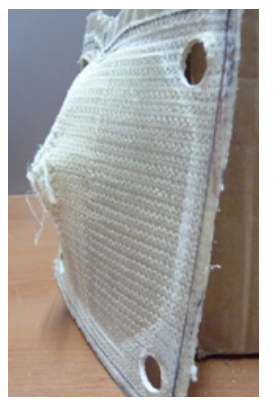

Fig. 4. Illustration of the back-face deformation after impact case of "Ortho2".

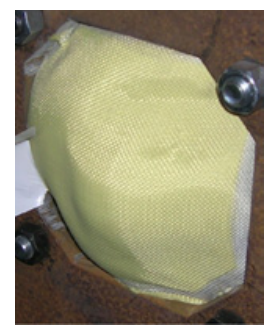

Fig. 5. Illustration of the back-face deformation after impact case of "15 plies Prepreg".

(Table 5). However, when we modify the resin process, we can remark that, the warp interlocks structures have the same behaviour than prepreg. Indeed the impact ratio of "TtT2" interlock is near the Impact ratio of prepreg. The same observation could be made with the back-face deformation after impact, where the deformation of the "TtT2" interlock is near to the prepreg deformation leading to have equivalent Impact ratio. See also the illustration of the back-face deformation on the next figures 3, 4 and 5 .

\section{Conclusion}

This study helps us to understand the mechanical behaviour of warp interlocks structures during ballistic impact. The warp interlock weaving represents a large work dealing with different parameters as the geometrical structures, the density of yarns, and the nature of yarns.... All of these parameters influence the final behaviour of the material during the impact. Beyond the geometrical structure, we have noticed the influence of the resin process which largely influences the resin rate inside the structures and the deformation behaviour during the impact tests.

In this study, we have tried to highlight two warp interlocks woven structures infused by two infusion process. However, when we compare with existing protection as prepreg and layered structures, we can remark that it still remains to have the best ballistic results and impact behaviour. However, the warp interlocks structures infused with high pressure show similar results then prepreg. The next work will be to make tomography scan, in order to observe the influence of high pressure on the geometrical structure of the warp interlocks fabric.

Moreover, for the future, it will be interesting to associate different materials in order to combine their mechanical properties. Indeed, it could be investigated to work with plain weave fabrics or prepreg in association with warp interlocks structures. It will combine the deformation properties of 2D fabric and the 3D reinforcement of warp interlocks weaving structures. This idea is in progress in our laboratory and results will be soon available.

\section{Acknowledgements}

We would like to acknowledge the DGA and Nexter Systems for their kind help to carry out the study in good conditions.

\section{References}

1. "Behaviour of warp interlocks structures under an IEDs attack, application with orthogonal and throughthe-thickness interlocks", Lefebvre Marie, Boussu François, NATO ASI Defence Related Intelligent Textiles and Clothing for Ballistic and NBC (Nuclear, Biological, Chemical) Protection, Split (Croatie), April 2010

2. "Influence of warp interlock parameters on the ballistic behaviour of a structure", M. Lefebvre, F. Boussu, D. Coutellier, TEXCOMP 2010, International Conference on Textile Composites, Lille (France), 26-28 Octobre 2010, ISBN 978-1-6095-026-6

3. "Study of impact behaviour of three warp interlock structures. Comparison with existing protections." M. Lefebvre, François Boussu, Daniel Coutellier, LWAG 2011, Light-Weight Armour for Defence \& Security, Haifa (Israel), 16-17 Mars 2011

4. "Study of Impact behaviour of three warp interlock structures for armoured vehicle." M. Lefebvre, François Boussu, Daniel Coutellier, Daniel Vallee, Workshop on Dynamic Failure of Composites and Sandwich Structures, Juin 2011, Toulouse, (France)

5. Marie Lefebvre, François Boussu, Daniel Coutellier, «Influence des paramètres de tissage sur des structures interlock soumises à l'impact balistique » proceeding of the JNC, Journées Nationales Composites, Poitiers Juin 2011, ISBN 978-274-6634-1-83

6. Marie Lefebvre, François Boussu, Daniel Coutellier, « Degradation measurement of fibrous reinforcement inside composite material », Proceeding of ICCE 19, July 2011

7. S. Rudov-Clark, A.P. Mouritz, L. Lee, M.K. Bannister. Fibre damage in the manufacture of advanced three dimensional woven composites. Composites part A 2003; 34: 963-970 
8. On the comparison of the ballistic response of coated aluminum plates, Evren Ozsahin, Suleyman Tolun, Materials and Design 31 (2010) 3188-3193

9. Ballistic studies on layered structures, P.K. Jena, K. Ramanjeneyulu, K. Siva Kumar, T. Balakrishna Bhat Materials and Design 30 (2009) 1922-1929

10. Ballistic impact performance of composite structures, N. Tarim, F. Findik, H. Uzun, Composite Structures 56 (2002) 13-20

11. Siow YP, Shim VPW. An experimental study of low velocity impact damage in woven fiber composites. J Compos Mater 1998;32:1178-202

12. Davies GAO, Hitchings D, Zhou G. Impact damage and residual strengths of woven fabric glass/ polyester laminates. Compos Part A 1996;27A:114756

13. Rydin RW, Bushman MB, Karbhari VM. The influence of velocity in low velocity impact testing of composites using the drop weight impact tower. J Reinforced Plastics Compos 1995; 14:113-27
14. Wu E, Chang LC. Loading rate effect on woven glass laminated plates by penetration force. J Compos Mater 1998;32:702-21

15. Kim J-K, Sham M-L. Impact and delamination failure of wovenfabric composites. Compos Sci Technol 2000;60:745-63

16. Cox BN, Dadkhah MS. Macroscopic elasticity of 3D woven composites. J Compos Mater 1995;29(6):785819

17. Shoshanna D. Rudov-Clark, "Experimental Investigation of the tensile properties and failure mechanism of three-dimensional woven composites", School of Aerospace Mechanical and Manufacturing Engineering, RMIT University, PhD thesis March 2007

18. Julian HU, "3D fibrous assemblies, properties applications and modelling of three dimensional textile structures", page 7, WP number 74, ISBN 978-184569-377-0, 2008

19. Wisetex software, developped by S. Lomov, KU Leuven University 